\title{
Hybrid Optimization for Wind Turbine Thick Airfoils
}

\author{
Francesco Grasso
}

Presented at the the $8^{\text {th }}$ AIAA Multidisciplinary Design Optimization Specialist Conference, 23-26 April 2012, Honolulu, HI, USA 


\title{
Hybrid Optimization for Wind Turbine Thick Airfoils
}

\author{
F. Grasso ${ }^{1}$ \\ Energy research Centre of the Netherlands (ECN), Petten, the Netherlands, 1755LE
}

\begin{abstract}
One important element in aerodynamic design of wind turbines is the use of specially tailored airfoils to increase the ratio of energy capture and reduce cost of energy. This work is focused on the design of thick airfoils for wind turbines by using numerical optimization. A hybrid scheme is proposed in which genetic and gradient based algorithms are combined together to improve the accuracy and the reliability of the design. Firstly, the requirements and the constraints for this class of airfoils are described; then, the hybrid approach is presented. The final part of this work is dedicated to illustrate a numerical example regarding the design of a new thick airfoil. The results are discussed and compared to existing airfoils.
\end{abstract}

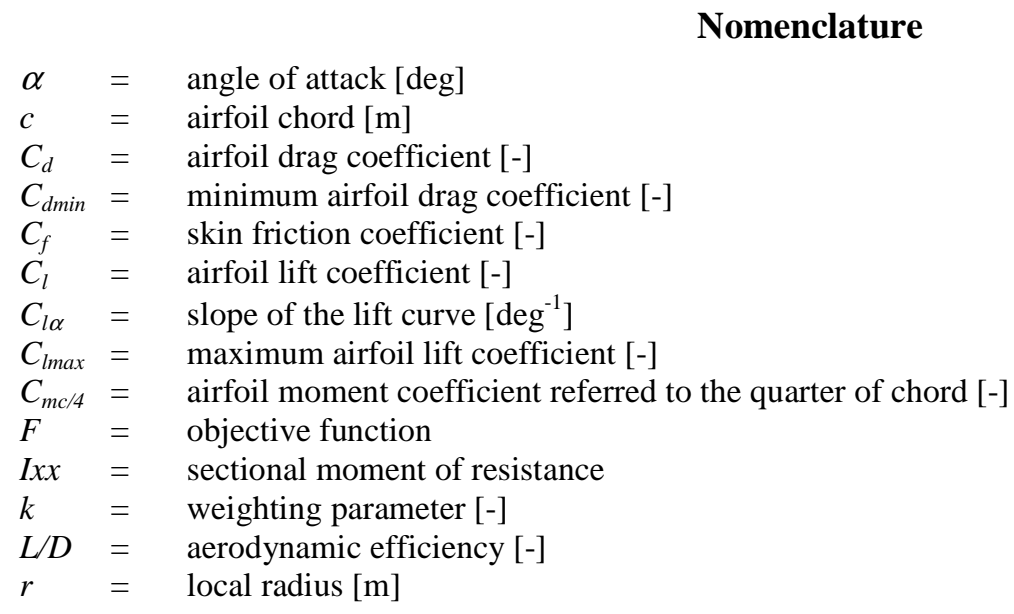

\section{Introduction}

$\mathrm{D}$

ESIGN of airfoils specifically suited for wind turbine blade applications is important in the continuing development of wind turbines. Because of the intrinsic requirements in terms of design point, off-design capabilities and structural properties, new airfoils dedicated to wind turbine application have been developed ${ }^{1-4}$. However, most of these airfoils were designed for the outer part of the blade where the aerodynamic requirements have higher priority compared to the structural ones.

Timmer $^{4}$, Hoerner ${ }^{5,6}$, van Dam, Mayda and $\mathrm{Chao}^{7}$ made significant research on thick airfoils. Nowadays, the design of geometries with relatively large trailing edge thickness (blunt trailing edge or flat-back airfoils) became popular in order to improve the airfoil's lift performance.

From numerical point of view, this class of geometries is challenging, due to their intrinsic characteristics and the lack of experimental data to validate the numerical predictions. Also, the fact that aerodynamic performance should be conjugated together with structural properties, makes the problem even more attractive from the design point of view.

In the last decades, multidisciplinary design optimization (MDO) became more and more attractive and effective approach instead of traditional methodologies during the design process. This is due to the rapid increase in computational resources normally available, but also to the fact that the algorithms are more efficient and robust, giving the opportunity to the designers to include several disciplines in their procedures.

\footnotetext{
1 Postdoctoral Aerodynamicist, Wind Energy Unit, Wind Energy Technology Group, Westerduinweg 3; grasso@ecn.nl, Associate Fellow AIAA.
} 
In the next section, the requirements for this class of airfoils are presented, then the used approach is explained. Finally, the design of the new airfoil is described and the results are discussed.

\section{Airfoils for the Inner Part of the Blade}

Airfoil characteristics include both aerodynamic and structural requirements. For the inner part of the blade, the structural requirements have higher priority than for the sections in the outer part of the blade. In order to guarantee the needed structural strength and stiffness, the airfoils at the root have large values of moment of resistance; this means large values of thickness and sectional area. In this perspective, flat back airfoils are helpful to improve the structural properties.

From the aerodynamic point of view the most important parameter for the tip region is the aerodynamic efficiency $(L / D)$. In order to obtain good turbine performance, the aerodynamic efficiency should be as high as possible, but, at the same time, other considerations should be taken into account.

For the inner part of the blades, the lift coefficient $\left(C_{l}\right)$ itself is very important as well as the stall behaviour. This is because in normal operating conditions, the local angle of attack can be quite high. Good values of $C_{\text {lmax }}$ and good stall characteristics can help to keep high aerodynamic performance and prevent structural problems for the blade.

Another consideration is related to presence of gusts. The local angle of attack can rapidly change, so there should be a "safety" margin between the design angle of attack and the stall angle in order to avoid that the airfoil works in stall or across the stall region that can lead to fatigue problems.

Also, wind turbines should be efficient in dirty conditions as well; this means that the sections need to be insensitive to roughness.

For the outer part of the blade, the connection between aerodynamic performance and structural response of the blade (i.e. blade torsion) is a crucial element during the design; for the inner part, this connection should be still taken into account, even if with lower priority. A complete discussion about requirements for wind turbine airfoils can be found in Ref. 8 by the present author.

\section{Design Approach}

Hoerner and van Dam proposed a way to generate thick airfoils with blunt trailing edge (by systematically cutting part of the trailing edge region according to Hoerner, or by smoothly increasing the thickness at the rear part of the airfoil according to van Dam.). However, in general both procedures cannot be used as design procedure in the sense that the characteristics of the final airfoil cannot be fully controlled and these characteristics are really dependent on those ones of the initial geometry. As mentioned, for this class of airfoils the structural characteristics are playing an important role for their real usage, so a cut-and-try approach can lead to not optimal solutions.

In the present work, a numerical optimization based approach has been used in order to have an efficient design method able to deal with multiple requirements coming from different disciplines.

\section{A. Optimization Scheme}

Nowadays, several algorithms are available for optimization, belonging to different categories depending on the mathematical formulation. Usually, evolutionary algorithms are less sensitive to local minima, but they are time consuming and constraints have to be included as a penalty term to the objective function. On the other hand, gradient based algorithms (GBA) can lack in global optimality but allow multiple constraints and are more robust, especially for problems in which a large number of constraints are prescribed. In this research, a hybrid scheme has been implemented in which genetic algorithms (GA) and GBA are combined together to improve the accuracy and the reliability of the design.

GA are used at the beginning of the design process in order to explore large domain with less problems regarding local optima. The optimal solution found by the GA is then used as initial configuration for the GBA that aims to reach a more accurate optimal solution in a smaller design space. Despite the fact that a single design procedure can be implemented in which automatically the result of GA is further improved by GBA, the two algorithms are kept independent from each other. Separate runs are necessary to complete a single design. The reason of this choice is that GBA can be sensitive to the initial geometry and a sub-optimal solution from GA could lead to a better final solution. One of the goals of the present work is also to investigate what is the best combination of GA and GBA in order to have the best final solution but also to reduce the computational time.

The GBA implemented in this work is the sequential quadratic progrmming method developed by Schittkowsky ${ }^{9}$, with the gradients approximated by finite differences.

Regarding the GA, the algorithm developed by Yang, Reinstein, Pai, Xu, and Carroll ${ }^{10}$ has been preferred. Tournament selection scheme has been used, together with uniform crossover and elitism mechanisms. Also, both 
jump mutation and creep mutation have been activated. One of the unique characteristics of Carroll's GA is the so called "MicroGA"; it is a method to improve the performance of genetic algorithms used in this work and derived by the studies of Goldberg and Krishnakumar ${ }^{11}$ to explore the use of small population sizes in genetics. Small populations can lead to too rapid convergence, so the re-generation of random population members helps to ensure diversity during the search process and so to avoid local minima.

For the implementation of the constraints in GA, a zero value is assigned to the objective function in case at least one constraint is violated.

\section{B. Geometry Parameterization}

A composite cubic Bezier parameterization is used, based on the development done by the present author ${ }^{13}$. The design variables of this parameterization are the coordinates of the control points (see figure 1).

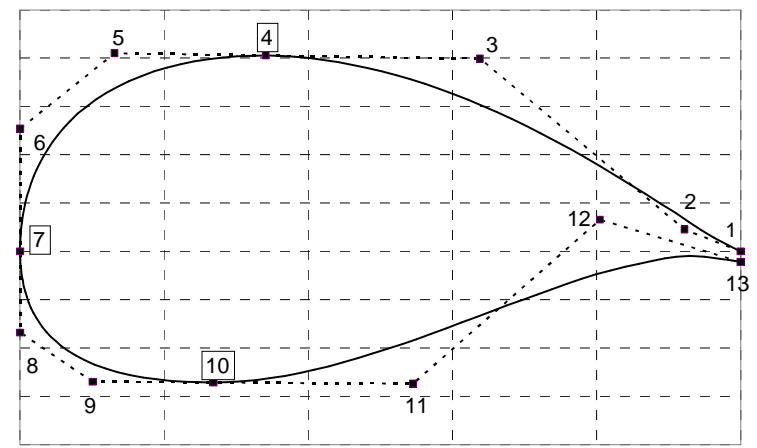

Figure 1 Sketch of the parameterization.

\section{Objective Function Definition and Evaluation}

Aerodynamic efficiency has been used as aerodynamic performance to be improved, together with the sectional moment of resistance for the structural part of the problem. These two requirements are conflicting with each other; in fact the aerodynamic efficiency is pushing the design to thin geometries, while the structural requirements would prefer thick shapes. The two objectives are combined together in form of a weighted linear combination (see eq. 1); this means that the final shape is also function of the relative importance of the aerodynamics w.r.t. the structures. A non dimensional parameter $k$ is used to define the relative importance of the two contributions to the global objective function. In order to create a family of new airfoils and investigate the effect due to the value of $k$, the optimization process has been repeated several times by changing the value of the parameter $k$.

$$
F=k(L / D)+(1-k) I_{x x} \quad \text { eq. } 1
$$

Where $\mathrm{k}$ is a weighting parameter varying between 0 and 1 .

The sectional inertia moment is depending only on the airfoil's geometry but an aerodynamic solver has been necessary to evaluate the aerodynamic efficiency (and some aerodynamic constraints, see next section for details). RFOIL $^{14}$ code has been used.

RFOIL is a modified version of XFOIL ${ }^{15}$ featuring an improved prediction around the maximum lift coefficient and capabilities of predicting the effect of rotation on airfoil characteristics.

In order to assess the accuracy of RFOIL for this particular class of geometries, DU00-W2-350, DU-00-W2-401, FX77-W-343 and FX77-W-400 airfoils have been used as reference to validate the numerical predictions. The Delft University (DU) airfoils have been tested at Delft University, the FX airfoils instead at Stuttgart University. All the tests have been performed at 3 million Reynolds number. 

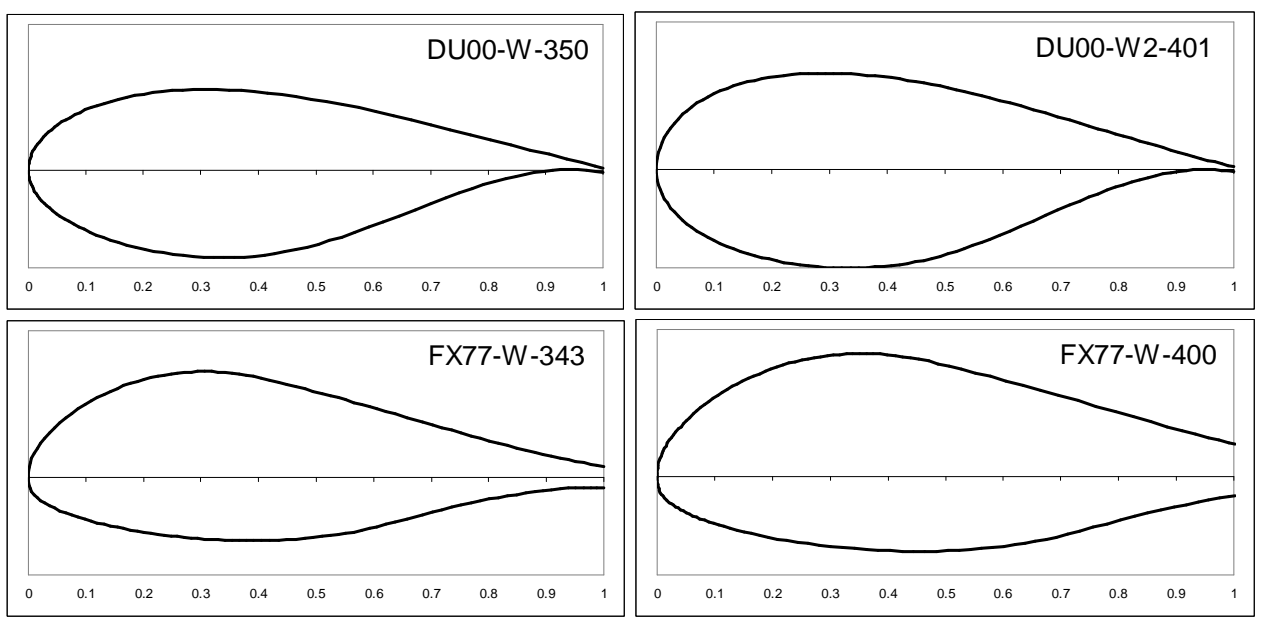

Figure 2 Airfoils selected for the validation.

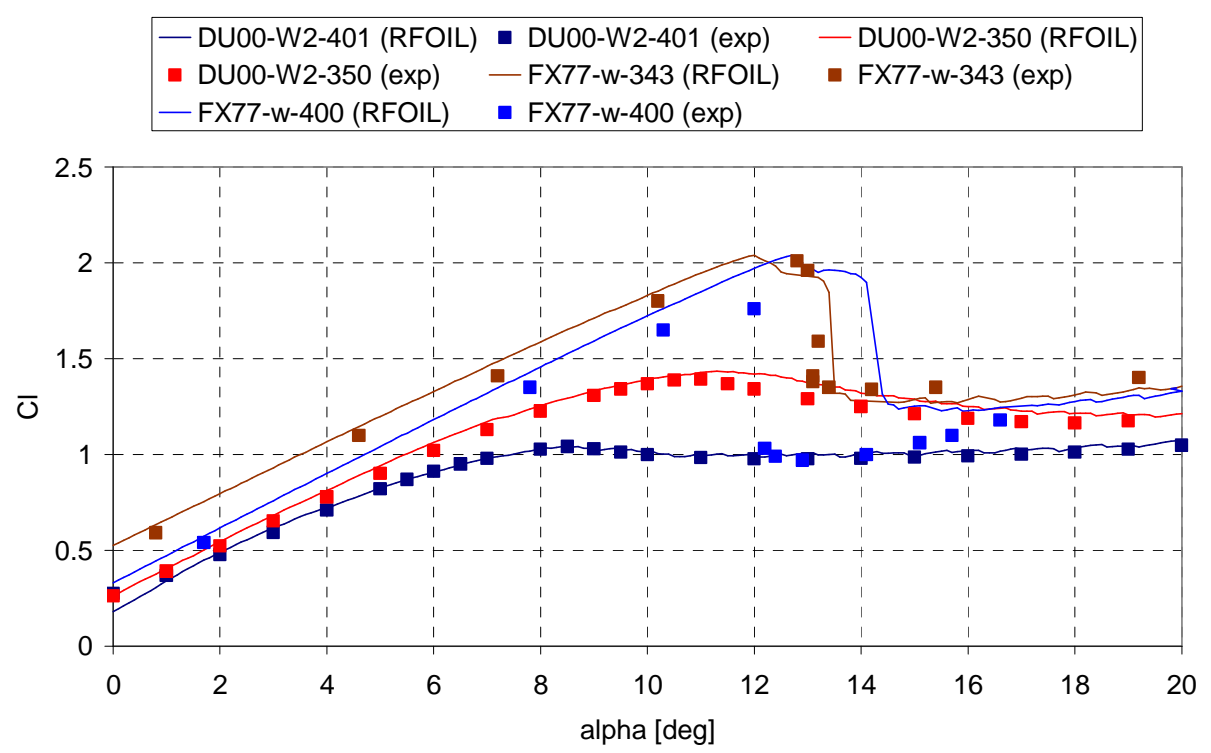

Figure 3 Validation for thick airfoils; lift curve. Reynolds number: 3 million. Experimental data from Refs. 4 and 16.

For the DU airfoils the agreement in terms of lift performance is really good; in terms of drag, an underestimation around $13 \%$ has been found, which is in line with the results of previous validations done on thinner geometries. Looking at the FX airfoils, the lift curve is quite well described, at the least the general shape of the curve. For the FX77-W-400 there is an overestimation of the maximum lift coefficient and consequently of the angle of stall, but the abrupt shape of the stall is anyway captured. In terms of drag instead, the underestimation is higher, especially for the $40 \%$ airfoil. The reason of that can be related with lack of accuracy in presence of large trailing edge thickness. 

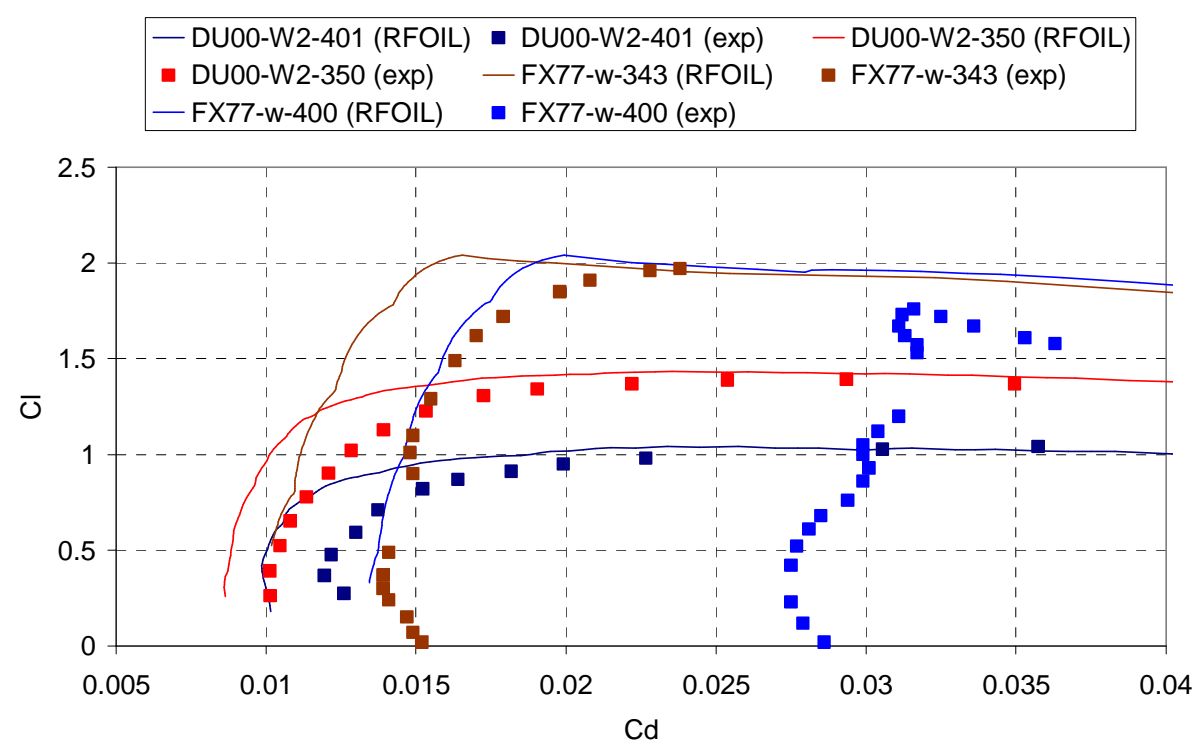

Figure 4 Validation for thick airfoils; polar curve. Reynolds number: 3 million. Experimental data from Refs. 4 and 16.

\section{Design of New Thick Airfoils}

\section{A. Definition of the Requirements and Constraints}

The design of new airfoil for the inner part of the blade is presented in this section. The goal of the optimization process is to maximize the aerodynamic efficiency and the moment of resistance at the same time. As final result, a family of airfoils should be expected, with the relative thickness increasing, depending on the structural needs.

The Reynolds number during the design is 3 million (intended to be representative of a MW class wind turbine) and the design angle of attack is 6 degrees. Also, the design is performed by imposing the transition at $1 \%$ of the chord on the suction side and $10 \%$ for the pressure side. The reason of that is to obtain airfoils wich exhibit a good insensitivity to the roughness by driving the design process to geometries without large extent of laminar flow.

From geometrical point of view, a minimum airfoil thickness of $35 \%$ of the chord and a minimum thickness at the trailing edge of $1 \%$ of the chord have been prescribed. The constraint related to the trailing edge thickness has been assigned to take into account manufacturing requirements; an upper bound for this variable is not prescribed, $\mathrm{n}$ order to be able to explore flat-back solutions.

In addition to these geometrical constraints, several aerodynamic constraints have been defined. As discussed in section II, the effect of aerodynamic performance on structural deformations should be taken into account; in particular, the torsion of the blade has strong impact on selection of the materials and costs. Also, considering that several airfoils are installed on the same blade, the aerodynamic properties of the single airfoils shouldn't differ too much between each other (see Ref. 8). For these reasons, a maximum value for the moment coefficient $\left(C_{m c / 4}\right)$ equal to -0.15 has been assigned at the design condition ( 6 degrees of angle of attack).

In normal operating conditions, the sections at the root of the blade work at high angle of attack; this implies that the airfoils need to be designed considering the stall condition and the airfoil's separation point movement as part of the core requirements. In fact, good $C_{l \max }$ values should be achievable, in conjunction with soft stall and post-stall characteristics.

Also, a margin between design condition and stall condition should be kept to take into account the angle of attack change due to gusts. In the reference mentioned above, an explicit constraint on the position of the separation point was used; in this research, however, a different approach has been preferred. Two combined constraints have been implemented to control the stall characteristics of the airfoils. The first constraint consists of imposing a value of $C_{l}$ lower than 1.8 at 15 degrees of angle of attack; the second constraint consists of imposing that the drop in $C_{l}$ between 15 and 16 degrees is less than 0.3. The effect of these two combined constraints should be to avoid excessive high lift performance that could lead to an abrupt stall. The value of 15 degrees has been selected taking into account some margin from the design condition; 1.8 as value for $C_{l}$ is still high lift. Regarding the second 
constraint, the threshold value has been selected after comparison of experimental data from Abbott and von Doenhoff ${ }^{17}$ for 4,5 and 6 digits NACA airfoils.

\section{B. Design Variables}

Fifteen design variables have been actively used in this case, corresponding to (see figure 1) control points 2,3 , $5,9,11$ and 12 in both directions and control points $1,6,8$ only in vertical direction.

It should be noticed that keeping free the control point 1 , it allows the optimization scheme to search also in the space of blunt trailing edge airfoils. One of the drawbacks of the solution proposed by Hoerner to generate thick airfoils is the fact that the chord is not anymore horizontal, affecting the real angle of attack. To avoid this problem, the control point 13 is mirrored according to the value of the control point 1 .

In table 2, the values for upper and lower bounds are listed. According to these values, airfoils with thickness varying from $10 \%$ up to $50 \%$ of the chord can be explored. The trailing edge thickness can increase up to $20 \%$ of the chord.

\begin{tabular}{cccc}
\hline ID & $\begin{array}{c}\text { Control } \\
\text { point* }\end{array}$ & $\begin{array}{c}\text { Lower bound } \\
\text { (\%chord) }\end{array}$ & $\begin{array}{c}\text { Upper bound } \\
\text { (\%chord) }\end{array}$ \\
\hline 1 & $2 \mathrm{~h}$ & 0.7 & 0.8 \\
2 & $3 \mathrm{~h}$ & 0.4 & 0.5 \\
3 & $5 \mathrm{~h}$ & 0.09 & 0.15 \\
4 & $9 \mathrm{~h}$ & 0.09 & 0.15 \\
5 & $11 \mathrm{~h}$ & 0.4 & 0.5 \\
6 & $12 \mathrm{~h}$ & 0.7 & 0.8 \\
7 & $1 \mathrm{v}$ & 0 & 0.1 \\
8 & $2 \mathrm{v}$ & 0.03 & 0.2 \\
9 & $3 \mathrm{v}$ & 0.08 & 0.25 \\
10 & $5 \mathrm{v}$ & 0.08 & 0.25 \\
11 & $6 \mathrm{v}$ & 0.03 & 0.1 \\
12 & $8 \mathrm{v}$ & -0.1 & -0.02 \\
13 & $9 \mathrm{v}$ & -0.25 & -0.02 \\
14 & $11 \mathrm{v}$ & -0.25 & 0.04 \\
15 & $12 \mathrm{v}$ & -0.1 & 0.09 \\
\hline \hline
\end{tabular}

Table 1 Bounds for the design variables. " $h$ " denotes the horizontal direction, "v" the vertical direction.

\section{Preliminary Tests}

Preliminary investigations have been performed separately on GA and GBA. In this paragraph, some of the results found in these tests are illustrated. A simplified version of the optimization problem presented in the previous section has been used; the objective function is just the aerodynamic efficiency at 6 degrees of angle of attack. It should be also underlined that look only at one parameter is not enough to decide about the quality of an airfoil, since many characteristics, often conflicting with each other, should be evaluated. Usually a good airfoil is a combination of several characteristics. However, in this context, the interest is focused on the response of the algorithms, more than the real airfoil design problem. All the tests have been performed on a machine with Centrino-Duo processor (CPU@2Ghz).

\section{Genetic Algorithm}

As mentioned, the used GA has the special microGA scheme. The tests have been focused on evaluate the effects of such scheme w.r.t. the traditional scheme; also, the influence of the population size, number of generations and children per pair, have been considered. Population size of 5 and 10 individuals have been used, evolving up to 400 generations with and without microGA scheme. With microGA, only 1 child is allowed per pair; instead, without it, 1 and 2 children are allowed.

Finally, both a complete set of constraints and a reduced one have been used; in particular, the constraints about the stall have been deactivated. The idea was to use a subset of constraints in GA to reduce the computational time, while in GBA the refinement is always performed with the full set of requirements. In principle, this could save some time and force the GBA to change the solution (so avoiding local optima) to satisfy the whole set of conditions. Tablexxx summarizes some of the results of these tests performed on the reduced set of constraints. 


\begin{tabular}{cccccc}
\hline \hline ID & microGA & population & children & Time $(\min )$ & L/D \\
\hline A & yes & 5 & 1 & 25 & 55.48 \\
B & yes & 10 & 1 & 130 & 68 \\
C & no & 5 & 1 & 43 & 62.1 \\
D & no & 5 & 2 & 30 & 52.4 \\
E & no & 10 & 1 & 150 & 60 \\
F & no & 10 & 2 & 70 & 57.7 \\
\hline \hline
\end{tabular}

Table 2 Summary of results for preliminary tests on GA (not complete set of constraints).

With reference to the table, the solutions for configurations B, C and E are the most promising; however, the airfoils generated for $\mathrm{B}$ and $\mathrm{E}$ showed a not completely regular shape on the suction side, leading to a very sharp stall. Also, they are the most expensive in terms of computational costs. From the design process however, this result can be avoided only by adding extra constraints on the stall, so using the complete set of constraints. With the complete set, the average computational time is increasing, since the simulations take longer, and the difference in results is reduced, due to the effect of the extra constraint. However, the trend does not change and the configuration $\mathrm{C}$ is still the best (table 4). It should be stressed that the fact that the stall is not assigned explicitily, does not mean that the stall is sharp.

\begin{tabular}{cccccc}
\hline \hline ID & microGA & population & children & Time $(\min )$ & L/D \\
\hline A & yes & 5 & 1 & 110 & 55.7 \\
B & yes & 10 & 1 & 130 & 55.7 \\
C & no & 5 & 1 & 170 & 56 \\
D & no & 5 & 2 & 120 & 53 \\
\hline \hline
\end{tabular}

Table 3 Summary of results for preliminary tests on GA (complete set of constraints).

According to the tables, very small populations with 1 child per pair produce better values. Figure 5 illustrates the evolution of the configurations in case of not complete set of constraints.

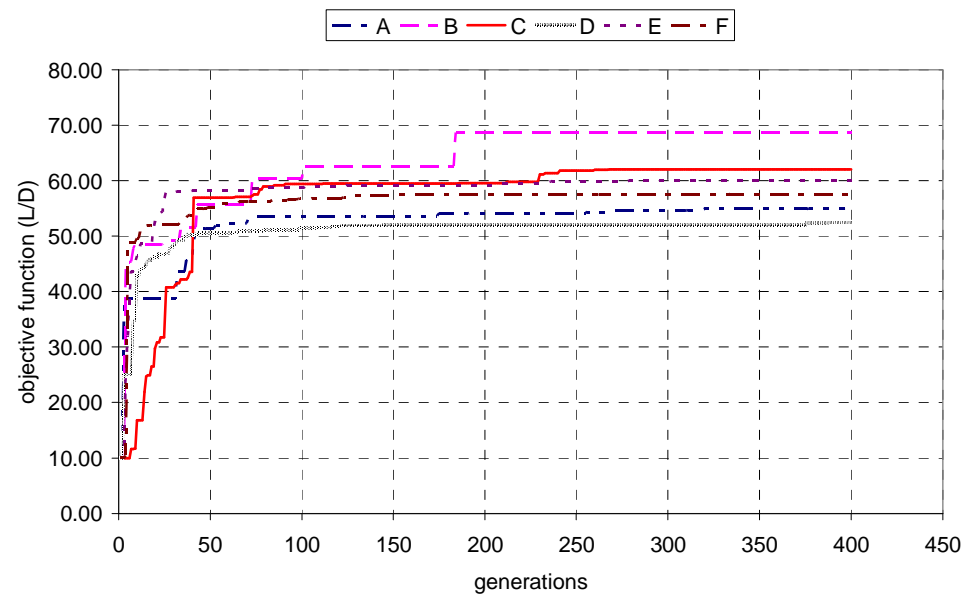

Figure 5 Best individual evolution.

\section{Gradient Based Algorithm}

The aim of the tests performed on GBA, was to assess the sensitivity of the algorithm to the initial solution and the sensitivity to local optima. Also, in order to have confidence on the hybrid optimization scheme, it was important to understand the response of the GBA depending on the GA individual used as baseline.

The configuration $\mathrm{C}$ has been used as reference and several optimizations with GBA have been performed by changing the generation where to consider ended the GA process. The best individuals from the generations 4,25 , 50, 100, 200 and 400 have been used; the results are shown in table 4. 


\begin{tabular}{cccccc}
\hline \hline \multirow{2}{*}{ ID } & $\begin{array}{c}\text { GA } \\
\text { generation }\end{array}$ & GA & GBA & $\Delta \%$ & T (sec) \\
\hline A1 & 400 & 55.48 & 60 & 8.14708 & 453 \\
C0 & 4 & 10 & 58 & 480 & 652 \\
C1 & 25 & 31.7 & 60 & 89.27445 & 767 \\
C2 & 50 & 56.9 & 56.9 & 0 & 935 \\
C3 & 100 & 59.4 & 59.42 & 0.03367 & 125 \\
C4 & 200 & 59.6 & 59.62 & 0.033557 & 128 \\
C5 & 400 & 62 & 62.05 & 0.080645 & 121 \\
\hline \hline
\end{tabular}

Table 4 Summary of results for preliminary tests on GBA.

Comparing the "C" solutions, it can be observed that the GBA is able to improve more when the starting point is at the beginning of the GA optimization; this is mostly due that the GBA has more freedom w.r.t. local optima found with GA. For G3, G4 and G5, the GBA is not able to move from the initial solution. Looking at the time, this increases according to the generation of the initial solution, so the GBA needs more time to find an improving path. According to these results, it is convenient to run the GBA after few iterations of GA in order to reduce the computational time and obtain an optimal result. In the table, also a solution obtained with microGA scheme has been used to perform the GBA, obtaining a very good solution. The indication coming from these analyses is that the best hybrid combinations are the one corresponding to few full GA generations or full microGA optimization before to start the GBA. In both cases, the computational time can be significantly reduced.

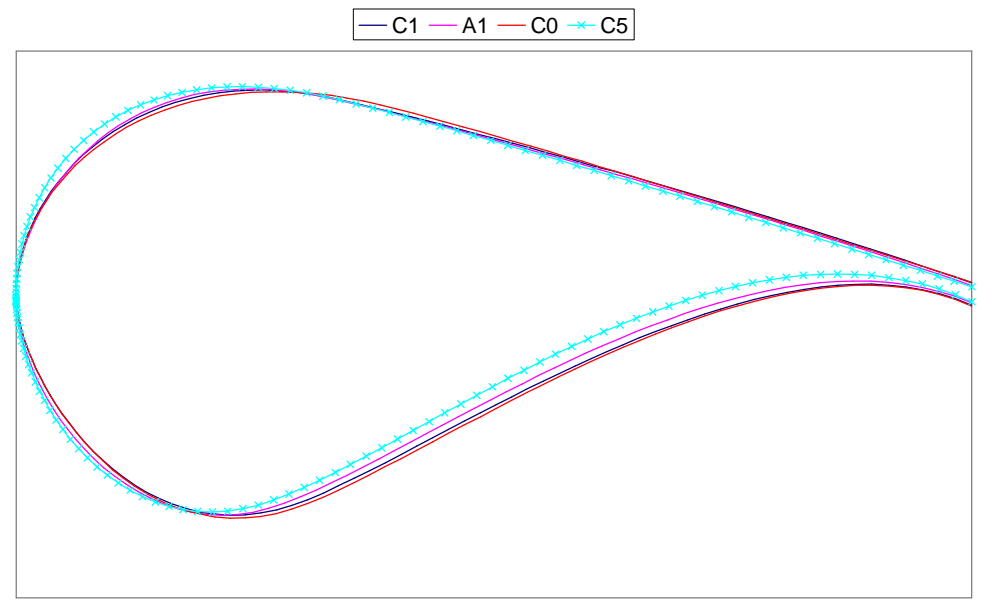

Figure 6 Comparison between the airfoil geometries.

\section{Design of Thick Airfoils}

In the present work, five individuals populations have been used, evolving for 200 generations. A summary of the used GA features can be found in table 5 .

\begin{tabular}{ccc} 
& Feature & Setting \\
\cline { 2 - 3 } Selection & Tournament \\
Crossover & Uniform \\
Mutation & Jump and Creep \\
Elitism & Active \\
MicroGA & Active \\
n. of parents & 2 \\
Population size & 5 \\
& Generations & 200 \\
\hline \hline
\end{tabular}

Table 5 Settings used for genetic algorithms. 
A family of new thick airfoils has been obtained by changing the relative importance of the aerodynamic requirements w.r.t. the structural requirements. The thickness varies between $50 \%$ of the chord for the geometries where the structural requirements were predominant (values of $k$ parameter close to 0 ), to $35 \%$ of the chord for the configurations where the aerodynamic efficiency was predominant (values of $k$ parameter close to 1). All the geometries exhibited blunt trailing edges, with larger values for the thicker airfoils. These first indications are consistent with the expectations because maximizing the moment of resistance means increasing the internal volume and viceversa for the aerodynamic efficiency. Figure 7 shows some of the developed geometries, figures 8 and 9 illustrate their aerodynamic characteristics.

In figure 8 and figure 9 also the characteristics of the DU00-W2-350 airfoil are illustrated. Compared to this geometry the new airfoils for $\mathrm{k}=0.8$ and $\mathrm{k}=0.9$ exhibit a higher value of $C_{l \max }$ and $\mathrm{L} / \mathrm{D}_{\max }$, together with a larger extension of the linear zone of the lift curve. This is positive to reduce fatigue problems due to gusts. In particular, the geometry for k=0.8 has also good stall characteristics with a value of $C_{m c / 4}$ close to the one of the reference geometry. The airfoils have been compared also in fixed transition; in this case, the performance of the new airfoils are evidently better than the rfeference geometry, both in terms of $C_{l}$ than $\mathrm{L} / \mathrm{D}$. It should be noticed the strange behaviour of the DU00-W2-350 airfoil, found also by van Rooij and Timmer ${ }^{18}$ during wind tunnel tests.

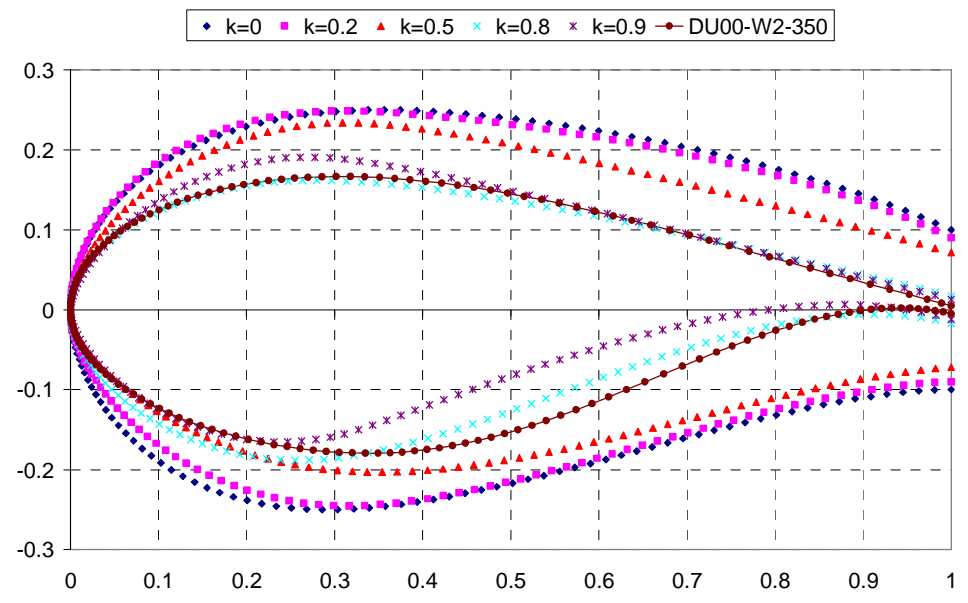

Figure 7 New geometries for different values of the weightin factor.

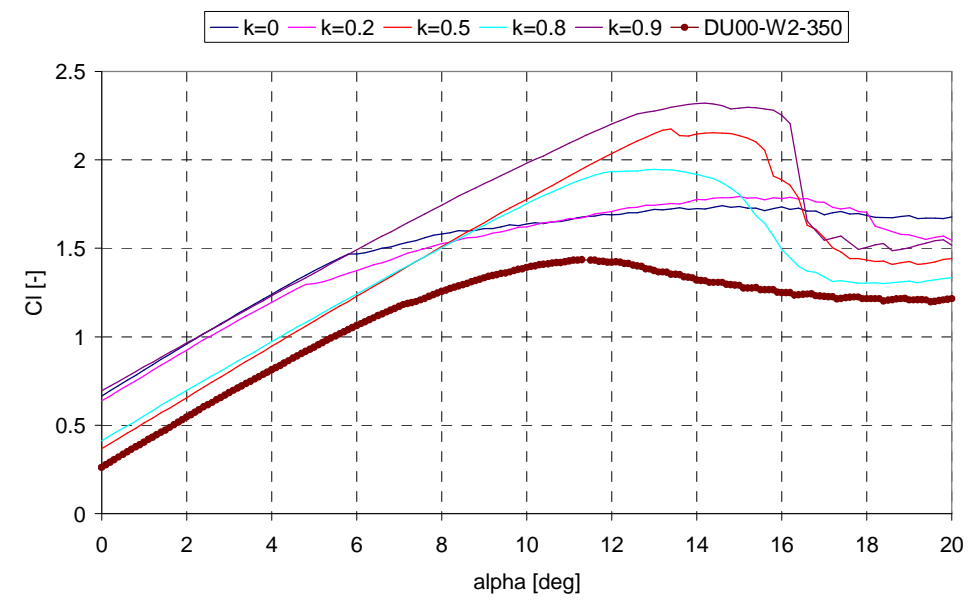

Figure 8 Lift curve for the new airfoils, compared to the DU00-W2-350 airfoil. Free transition, 3 million Reynolds number, RFOIL predictions. 


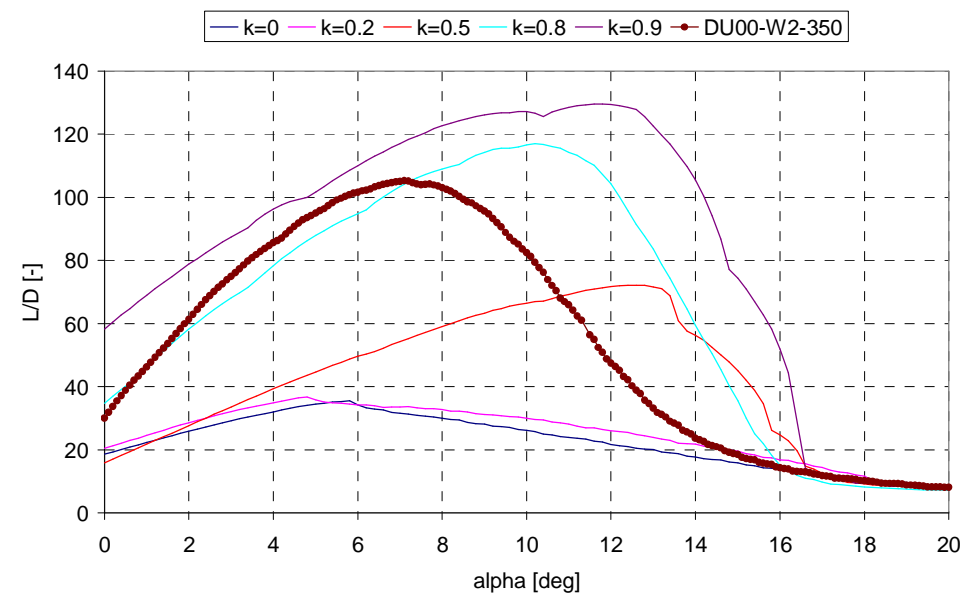

Figure 9 Efficiency curve for the new airfoils, compared to the DU00-W2-350 airfoil. Free transition, 3 million Reynolds number, RFOIL predictions.

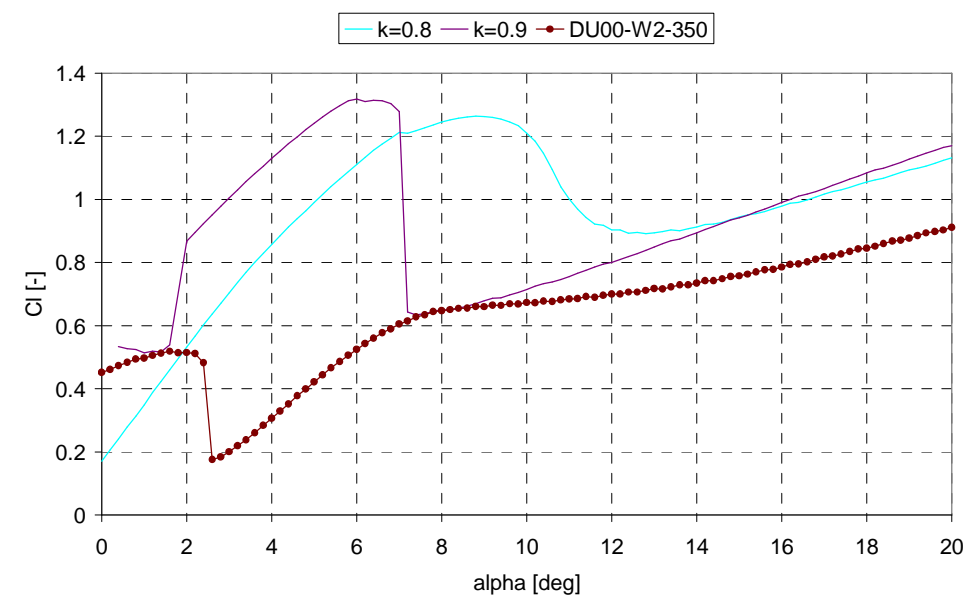

Figure 10 Lift curve for the new airfoils, compared to the DU00-W2-350 airfoil. Fixed transition, 3 million Reynolds number, RFOIL predictions.

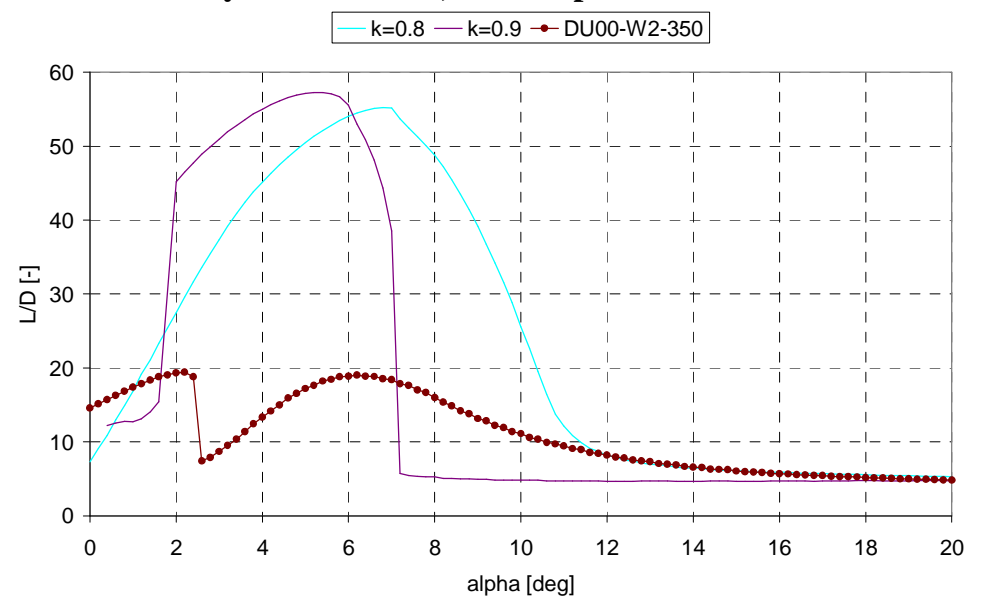

Figure 11 Efficiency curve for the new airfoils, compared to the DU00-W2-350 airfoil. Fixed transition, 3 million Reynolds number, RFOIL predictions. 


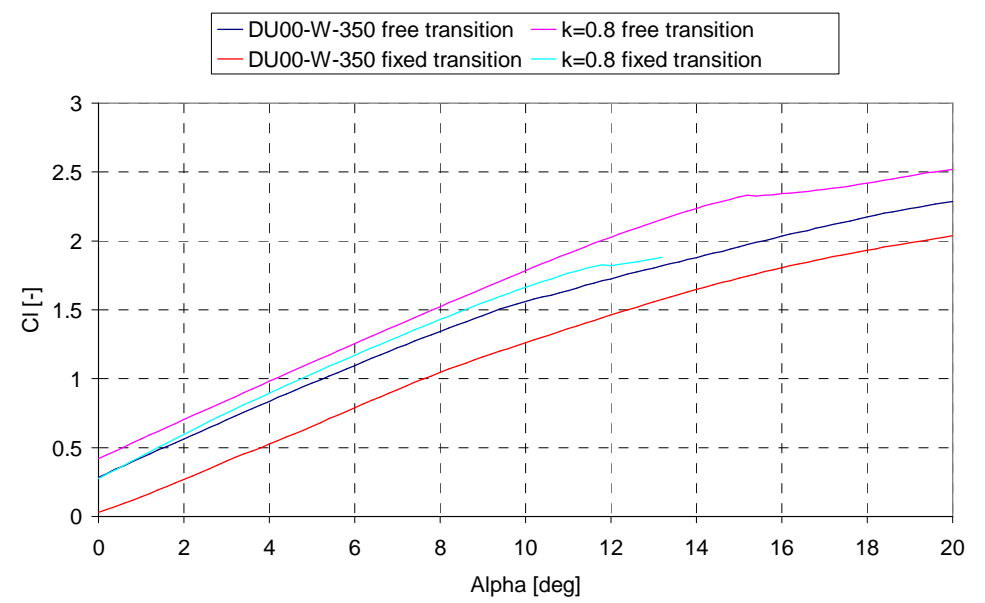

Figure 12 Lift curve in presence of rotational effects. 3million Reynolds number, free transition, $c / r=0.248$.

During the design, the rotational effects have not been taken into account, but at the root these effects are not negletable. Figure 12 shows the comparison between the lift curves in presence of rotational effects. These effects have been included in RFOIL by Snel, Houwink, van Bussel, and Bruining ${ }^{19}$. According to this formulation, the main parameter controlling the rottional effects is the ratio between local chord and local radius; a value of 0.248 for $\mathrm{c} / \mathrm{r}$ has been assigned. Both in free and in fixed transition, the new airfoil has better performance than the reference geometry. This is also due to the higher lift performance of the new airfoil.

In terms of optimization process, the combination of GA and GBA in a hybrid scheme produced good results, improving the reliability of the design and contributing to save computational time. In fact, in almost all the cases analyzed, the usage of GBA after GA made possible to achieve a better final solution. For some configurations however, the GBA was not able to change the solution coming from GA, meaning that probably the interaction between the two algorithms can still be improved.

\section{Conclusions}

A hybrid optimization scheme making use of genetic algorithms and gradient based algorithms has been developed and applied to design a new family of airfoils dedicated to the root region of the wind turbine blade. Both aerodynamic and structural requirements have been included in the optimization process and specific constraints have been implemented to control the stall behavior.

According to the numerical predictions, the results are promising, showing that the new airfoils have feasible shapes, consistent with the assigned requirements and with good performance. Despite these good results however, wind tunnel tests are mandatory to validate the numerical predictions, especially at the stall where the numerical accuracy can be lower.

For this class of airfoils, the rotational effects are playing an important role; in the next development, they will be included in the design process.

Regarding the hybrid approach, the results showed that the combination of genetic and gradient based algorithms can be beneficial to improve the accuracy and robustness of the design and reduce the computational time. However, improvements in constraints implementation in genetic algorithms and in general a better communication between two algorithms can lead to higher quality results.

\section{References}

1 Tangler, J.L., Somers, D.M., “NREL Airfoil Families for HAWT's”. Proc. WINDPOWER'95, Washington D.C., 1995; pp. $117-123$.

2 Björk, A., "Coordinates and Calculations for the FFA-W1-xxx, FFA-W2-xxx and FFA-W3-.xxx Series of Airfoils for Horizontal Axis Wind Turbines". FFA TN 1990-15, Stockholm, Sweden 1990.

3 Fuglsang, P., Bak, C., "Design and Verification of the new Ris $\varnothing$-A1 Airfoil Family for Wind Turbines". AIAA-2001-0028

4 Timmer, W.,A., van Rooij, R.P.J.O.M., "Summary of the Delft University Wind Turbine Dedicated Airfoils". AIAA-20030352 .

5 Hoerner, S.,F., “ Base Drag and Thick Trailing Edge”, Journal of the Aeronautical Sciences, Vol. 17, No. 10, Oct. 1950, pp. $622-628$. 
6 Hoerner, S. F., and Borst, H. V., Fluid-Dynamic Lift, Hoerner Fluid Dynamics, Bricktown, NJ, 1985, pp. 2-10, 2-11.

7 van Dam, C.P., Mayda, E.A., and Chao, D.D., "Computational Design and Analysis of Flatback Airfoil Wind Tunnel Experiments", SAND2008-1782, Sandia National Laboratories, Albuquerque, NM, March 2008.

8 Grasso, F., "Usage of Numerical Optimization in Wind Turbine Airfoil Design", 28th AIAA Applied Aerodynamics Conference, AIAA, Chicago, IL, 28 June-1 July, 2010. AIAA2010-4404. Also, AIAA, Journal of Aircraft, Vol.48, No.1, Jan.Feb. 2011, DOI: 10.2514/1.C031089.

9 Schittkowski, K., "NLPQLP: A new Fortran implementation of a sequential quadratic programming algorithm - user's guide, version 1.6", Report, Department of Mathematics, University of Bayreuth, 2001.

10 Yang, G., Reinstein, L.E., Pai, S., Xu, Z., and Carroll, D.L., "A new genetic algorithm technique in optimization of permanent 125-I prostate implants" Medical Physics, Vol. 25, No. 12, December 1998, pp. 2308-2315.

11 Krishnakumar, K.,"Micro-Genetic Algorithms for Stationary and Non-Stationary Function Optimization." SPIE: Intelligent Control and Adaptive Systems,1196, Philadelphia, PA, 1989.

12 Samareh, J., A., "Survey of Shape Parameterization Techniques for High-Fidelity Multidisciplinary Shape Optimization", AIAA Journal, Vol. 39, No. 5, May 2001, pp. 877-884.

13 Grasso, F., "Multi-Objective Numerical Optimization Applied to Aircraft Design", Ph.D. Thesis, Dip. Ingegneria Aerospaziale, Università di Napoli Federico II, Napoli, Italy, December 2008.

14 van Rooij, R.P.J.O.M., "Modification of the boundary layer calculation in RFOIL for improved airfoil stall prediction", Report IW-96087R TU-Delft, the Netherlands, September 1996.

15 Drela, M., "XFOIL 6.94 User Guide”, MIT Aero \& Astro, Dec 2001.

16 Althaus, D., "Stuttgarter Profilkatalog II: Niedriggeschwindigkeitsprofile", Vieweg\&Sohn Braunschweig, Stuttgart, 1996.

17 Abbott, I., Von Doenhoff, A., Theory of Wing Sections, Dover Publications, Inc., Dover edition, 1958.

18 van Rooij, R.P.J.O.M., Timmer, W.A., "Rougness Sensitivity Considerations for Thick Rotor Blade Airfoils", AIAA, AIAA 2003-0350, 2003.

19 Snel, H., Houwink, R., van Bussel, G.J.W., Bruining, A., 'Sectional Prediction of 3D Effects for Stalled Flow on Rotating Blades and Comparison with Measurements', Proc. European Community Wind Energy Conference, Lübeck-Travemünde, Germany, 8-12 March, 1993, pp. 395-399, H.S. Stephens \& Associates 